KINDER- UND JUGENDLITERATURFORSCHUNG 1997/98 


\section{Kinder- und \\ Jugendliteraturforschung 1997/98}

Mit einer Gesamtbibliographie

der Veröffentlichungen des Jahres 1997

In Zusammenarbeit mit der Arbeitsgemeinschaft

Kinder- und Jugendliteraturforschung und

in Verbindung mit Carola Pohlmann (Berlin),

Verena Rutschmann (Zürich), Ernst Seibert (Wien)

und Jack Zipes (Minneapolis)

herausgegeben von

Hans-Heino Ewers, Ulrich Nassen, Karin Richter und Rüdiger Steinlein

\section{Verlag J. B. Metzler Stuttgart - Weimar}


Redaktion: Gina Weinkauff, Hans-Heino Ewers (verantwortlich)

Rezensionen: Ulrich Nassen

Bibliographie: Karin Laubrecht

Layout: Hongquin Flicker

Der Druck dieses Jahrbuchs wurde durch die Arbeitsgemeinschaft Kinder- und Jugendliteraturforschung gefördert.

Die Deutsche Bibliothek - CIP-Einheitsaufnahme

Kinder- und Jugendliteraturforschung . . . :

mit einer Gesamtbibliographie der Veröffentlichungen des Jahres . . I

in Zusammenarbeit mit der Arbeitsgemeinschaft Kinder- und

Jugendliteraturforschung. - Stuttgart ; Weimar : Metzler.

Erscheint jährl. - Aufnahme nach 1994/95(1995)

1997/98 (1998) -

ISBN 978-3-476-01625-6

ISBN 978-3-476-03754-1 (eBook)

DOI 10.1007/978-3-476-03754-1

Dieses Werk einschließlich aller seiner Teile ist urheberrechtlich geschützt. Jede Verwertung außerhalb der engen Grenzen des Urheberrechtsgesetzes ist ohne Zustimmung des Verlages unzulässig und strafbar. Das gilt insbesondere für Vervielfältigungen, Übersetzungen, Mikroverfilmungen und die Einspeicherung und Verarbeitung in elektronischen Systemen.

(C) 1999 Springer-Verlag GmbH Deutschland Ursprünglich erschienen bei J. B. Metzlersche Verlagsbuchhandlung und Carl Ernst Poeschel Verlag GmbH in Stuttgart 1999 


\section{Inhalt}

\section{Berichte}

Andreas Bode: Kinder- und Jugendliteratur des 16.-20. Jahrhunderts. Die Sammlung Schulz in der Internationalen Jugendbibliothek (München) ...3.-. Carola Pohlmann: Die Sammlung der Originalillustrationen in der Staatsbibliothek zu Berlin ...6.-. Verena Rutschmann: Zwei Ausstellungen im Schweizerischen Jugendbuch-Institut: Meta Heusser-Schweizer (1797-1876) und Kurt Kläber/Held (1897-1959) ...8.-. Horst Heidtmann: Das Institut für angewandte Kindermedienforschung (IfaK) an der Hochschule für Bibliotheks- und Informationswesen - Fachhochschule Stuttgart ...11.-. Inge Wild: Familienszenen in der Kinder- und Jugendliteratur. Zehnte Jahrestagung der AG Kinder- und Jugendliteraturforschung vom 29. - 31. Mai 1997 in Kronberg/Taunus ... 15

\section{Beiträge}

\section{John Stephens}

Ideologie und narrativer Diskurs in Kinderbüchern

Ines-Bianca Vogdt

...mit den Worten des Kindes: Kinderlyrik und Lyrik der Moderne

\section{Ernst Seibert}

Einfachheit und Komplexität in der Literatur Marlen Haushofers

Gunter Reiß

Trödelmarkt der Träume.

Wilfried Hillers Musiktheater für Kinder nach Texten von Michael Ende

Miriam Schulte

Popkultur im Jugendroman.

Stil, Spaß und Subversion zwischen teen spirit und Pädagogik

\section{Forschungsberichte}

\section{Andrea Weinmann}

Die bundesrepublikanische und österreichische Kinderliteratur der Nachkriegszeit in der Literaturgeschichtsschreibung ....

\section{Dieter Petzold}

Kinder- und Jugendliteraturforschung in der deutschen und auswärtigen Anglistik 


\section{Vorwort}

Mit der Vorlage der vierten Folge dieses Jahrbuches können und wollen die Herausgeber nicht verschweigen, wie schwierig sich in Zeiten knapper werdender Bibliotheksetats Start und Etablierung eines solchen Projekts ausnehmen. Das Jahrbuch der Kinder- und Jugendliteraturforschung bedarf auch jetzt noch der Unterstützung in materieller wie ideeller Hinsicht. Es kann darüber hinaus langfristig nur gedeihen, wenn es auch in angrenzenden Bereichen - in der Literatur- und Kunstdidaktik, der Pädagogik, der Kindheits- und der Jugendforschung etwa - Beachtung findet.

Wie wenig sich selbst in Fachkreisen der bibliographische Dienst des Jahrbuches herumgesprochen hat, sei hier an einigen Beispielen verdeutlicht. Das Jahrbuch verzeichnet in der ersten Folge 12, in der zweiten 6 und in der dritten 7 Titel zum Thema Gewalt/Rechtsradikalismus/Rassismus in der Kinder- und Jugendliteratur; die 1997 publizierten Artikel von Gabriele Haefs und Hartmut Jonas zum selben Thema erwähnen von den in den Jahren 1994 bis 1996 erschienenen 25 Beiträgen nicht einen einzigen. Franz Pöggelers materialreiche Darstellung der "Printmedien im Kinder- und Jugendleben des 18. und 19. Jahrhunderts" verfährt so, als habe es eine historische Kinder- und Jugendliteraturforschung nie gegeben. Die Annahme, daß die Kinder- und Jugendliteratur immer noch weitgehend unerforscht sei, mag hie und da noch ihre Berechtigung haben; mehr und mehr aber stellt sie nur noch eine bequeme Ausrede dar.

Die eine oder andere Rezension des Jahrbuchs hat mehr oder weniger heftige Reaktionen ausgelöst. Sollten einzelne als Polemik empfunden haben, was als solche sicherlich nicht gemeint war, so bedauern die Herausgeber dies sehr. Die Grenzlinie zwischen sachlicher Kritik und Polemik ist gelegentlich schwer zu ziehen; für den Betroffenen verläuft sie nur zu oft anders als für denjenigen, der Kritik ausübt. Keinesfalls aber sind diejenigen, die sich als Forscherinnen bzw. Forscher der Kinder- und Jugendliteratur widmen, allein schon deshalb sakrosankt; so große Freude über jede bzw. jeden herrscht, der sich auf das Feld der Kinder- und Jugendliteraturforschung begeben hat, es gilt auch hier der Kritik sich zu stellen.

In dieser Folge wird ein theoretischer Beitrag eines ausländischen Kollegen in deutscher Übersetzung abgedruckt - eine Praxis, die in den nächsten Folgen hoffentlich fortgeführt werden kann.

Wie bereits im vergangenen Jahr, versendet die Redaktion des Jahrbuchs an alle interessierten Bezieher kostenlos eine für IBM-kompatible PC-Systeme (MS-DOS/Windows) ausgelegte Datenbankversion der bisher veröffentlichten Jahresbibliographien (siehe Gutschein am Ende des Bandes). Abonnenten erhalten die Updates automatisch; für alle diejenigen, die bereits eine erste Version der Datenbank erhalten haben, gilt ein vereinfachtes Verfahren: ein Update wird gegen Einsendung der Originaldiskette mit den Jahresbibliographien 1994-1996 zugesandt.

Anschriften der Herausgeber:

Prof. Dr. Hans-Heino Ewers, Institut für Jugendbuchforschung, Myliusstr. 30, 60323 Frankfurt/M.

Prof. Dr. Ulrich Nassen, Institut für Germanistik der Universität, Augustusplatz 9, 04109 Leipzig

Prof. Dr. Karin Richter, Pädagogische Hochschule - Institut für Grundschulpädagogik und Kindheitsforschung, 99089 Erfurt

Prof. Dr. Rüdiger Steinlein, Humboldt-Universität, Philosophische Fakultät II, Schützenstr. 21, 10117 Berlin 